\title{
Synergies between curation and research in sample return missions.
}

\author{
AURORE HUTZLER ${ }^{1}$, JAMES CARPENTER ${ }^{1}$, TIMOTHY \\ HALTIGIN $^{1}$, RHIAN H. JONES ${ }^{2}$, GERHARD KMINEK ${ }^{1}$, \\ BERNARD MARTY ${ }^{3}$, FRANCESCA E. MCDONALD ${ }^{1}$, \\ CAROLINE L. SMITH ${ }^{4}$ AND KIMBERLY T. TAIT ${ }^{5}$ \\ ${ }^{1}$ ESA/ESTEC \\ ${ }^{2}$ The University of Manchester \\ ${ }^{3}$ Université de Lorraine \\ ${ }^{4}$ The Natural History Museum \\ ${ }^{5}$ Royal Ontario Museum \\ Presenting Author: aurore.hutzler@gmail.com
}

Sample return missions are an increasingly important part of planetary exploration and research for understanding the origin, composition, and evolutionary processes of the Solar System, as well as characterising planetary surfaces and future space materials in preparation for sustained space exploration including by humans. During the preparation and assembly phases of these missions, scientific researchers and curators commonly work together with engineers to ensure that the science goals of the mission are met and that the samples are returned in a pristine state, and with the necessary contamination knowledge. On Earth, the curation team processes the samples in preparation for use by the science community. Here, we advocate for synergistic work between the curation team and the science community to increase efficiency and maximize the science return after samples are back on Earth. Approaches to accomplish this synergy can be in terms of decision making as well as with technical and procedural innovations [1] for curation and allocation protocols. For example, Mars Sample Return (MSR) has ambitious science goals [2], but it is expected to only bring back a limited amount of material. In addition, MSR must adhere to planetary protection requirements.

To ensure the efficient use of samples during early sample characterisation and generation of a sample catalogue, the objective driven scientific investigations and the sample safety assessments (planetary protection) in the case of restricted samples, a pre-planned sample management plan [3] is needed that includes clear mandates for the various actors and constant interaction and feedback between the curation and research teams [4]. One possible model includes allocating samples to a consortium of institutions proposing a coordinated analysis plan, rather than isolated laboratories, to ensure efficiency and accountability.

With increasing interest for sample return missions from the community and from space agencies, and several sample return campaigns underway, finding new ways to better utilize the samples is a timely and exciting endeavour.

[1] McCubbin F.M. et al (2019) Spa.Sci.Rev. 215:48

[2] iMOST (2018) https://mepag.jpl.nasa.gov/reports.cfm

[3] MSPG (2019) https://mepag.jpl.nasa.gov/reports.cfm? expand $=\mathrm{mspg}$

[4] Haltigin T. et al (2018) Astrobiology 18:S1 